1. Suzuki, M. et al. Nature Cell Biol. 6, 861-871(2004).

2. Orlean, P. in The Molecular and Cellular Biology of the Yeast Saccharomyces (eds Pringle, J. R., Broach, J. R. \& Jones, E. W.) Vol. 3 (Cold Spring Harbor Laboratory, New York, 1997).

3. Lew, D. J. Curr. Opin. Cell Biol. 15, 648-653 (2003).
4. Fitch, I. et al. Mol. Biol. Cell 3, 805-818 (1992).

5. Breeden, L. L. Curr. Biol. 10, R586-R588 (2000).

6. Pic, A. et al. EMBO J. 19, 1-12 (2000)

7. Schroer, T. A. Annu. Rev. Cell Dev. Biol. 20, 759-779 (2004).

8. Adames, N. R. \& Cooper, J. A. J. Cell Biol. 149
863-874 (2000).

9. Lee, W.-L., Oberle, J. R. \& Cooper, J. A. J. Cell Biol. 160, 355-364 (2003).

10. Sheeman, B. et al. Curr. Biol. 13, 364-372 (2003).

11. Heinisch, J., Lorberg, A., Schmitz, H.-P. \& Jacoby, J. Mol. Microbiol. 32, 671-680 (1999).

\section{Stretching cell fate}

Skeletal muscle comprises bundles of multinucleated muscle cells, or myofibres. A remarkable feature of skeletal muscle is its ability to undergo rapid repair in the face of acute damage. This process is driven by a group of normally quiescent stem cells - known as 'satellite cells' - that are found beneath the basal lamina surrounding each myofibre. However, one unresolved question concerns how the pool of satellite cells itself is maintained. Three scenarios have been postulated: first, there are two populations of satellite cells, one that differentiates and another that maintains the pool; second, there is one homogeneous pool, from which cells adopt different fates according to specific environmental cues; third, satellite cells represent a committed myogenic precursor, and bona fide muscle stem cells are instead located within the muscle interstitium. In a new development, Peter Zammit et. al. (J. Cell Biol. 166, 347-357; 2004) suggest that the second scenario is correct by showing that although most dividing satellite cells eventually differentiate, a small proportion actually exit the differentiation programme and revert to a quiescent state.

The authors concentrate on two transcription factors, MyoD and Pax7, to follow cell fate. Expression of MyoD is associated with commitment to myogenic differentiation, whereas Pax7 expression is associated with satellite cell specification. One key aspect of the study was to culture freshly isolated intact mouse myofibres. In this way Zammit et al. aimed to recapitulate the in vivo situation more accurately than through culture of satellite cells alone, and to maintain any potential interactions between the satellite cells and the myofibre. In culture, almost all the originally quiescent satellite cells became activated and were found to express both MyoD and Pax7. Most cells proliferated, downregulated Pax7 and underwent terminal myogenic differentiation, often within large clusters of satellite cell progeny. Intriguingly, however, other satellite cells within these clusters adopted an alternative fate. Rather than undergoing differentiation, they downregulated expression of

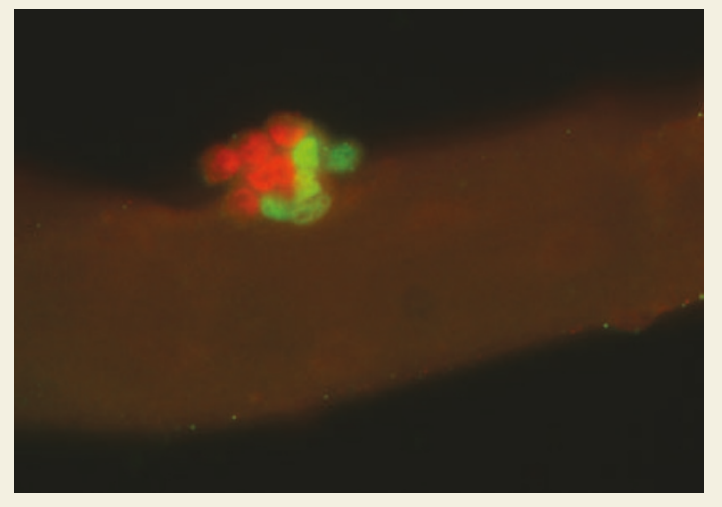

Immunostaining reveals divergent fates within a cluster of satellite cell progeny associated with an isolated myofibre. Most maintain MyoD (red) but lose Pax7 and differentiate. Others, however, maintain Pax7 (green) and downregulate MyoD, characteristic of quiescent satellite cells. This provides evidence of satellite cell self-renewal.

MyoD and became quiescent, maintaining the satellite cell pool. The authors also showed that these cells could not have arisen from a rapidly dividing pool of MyoD-negative, Pax7-positive, cells.

This result is interesting on two levels. On one, it addresses the long-standing question of how the satellite cell pool is maintained, suggesting that this occurs through adoption of divergent fates. On another, it challenges the widely held belief that MyoD expression commits cells to an irreversible programme of differentiation. One obvious conclusion, then, is that a mechanism exists to suppress MyoD expression. The next step will be to understand how MyoD downregulation occurs. A clue may come from the fact that this phenomenon is rarely observed in dispersed cultures of satellite cells maintained on plastic. This would suggest that the key to these events lies in interactions between satellite cells or with the underlying myofibre.

DONALD MCDONALD 